(2) Open Access Full Text Article

\title{
Potential role of enzastaurin in the treatment of patients with relapsed or refractory advanced cutaneous T-cell lymphomas: a review
}

This article was published in the following Dove Press journal:

Orphan Drugs: Research and Reviews

21 June 2012

Number of times this article has been viewed

\section{Deborah A Katz \\ Janet MD Plate \\ Sunita Nathan \\ Lydia Usha}

Division of Hematology and Oncology, Rush University Medical Center,

Chicago, IL, USA
Correspondence: Lydia Usha

Rush University Medical Center,

1725 West Harrison Street - Suite 809,

Chicago, IL 606I2, USA

Tel +I 3I25632200

$\mathrm{Fax}+13129423192$

Email lydia_usha@rush.edu
Abstract: Cutaneous T-cell lymphomas (CTCLs) are rare extranodal non-Hodgkin lymphomas characterized by neoplastic T-lymphocyte accumulation in the skin. The two most common types of CTCLs are mycosis fungoides and the leukemic variant, Sézary syndrome. Prognosis of CTCLs depends on the stage, with a poor prognosis in advanced-stage disease. A number of agents have recently been developed for the treatment of CTCLs: chemotherapeutic agents such as pralatrexate, interferon-alpha, retinoids such as bexarotene, monoclonal antibodies such as alemtuzumab, and histone deacetylase inhibitors such as vorinostat and romidepsin. Nevertheless, there is no cure for CTCLs except for allogeneic stem cell transplant. A promising new drug is enzastaurin. Enzastaurin is a novel serine/threonine kinase inhibitor that binds to protein kinase $C-\beta$ (PKC- $\beta$ ) and inhibits the phosphoinositide- 3 kinase (PI3K)/AKT/phosphatase and tensin homolog (PTEN) signaling pathway. Enzastaurin induces apoptosis and inhibits angiogenesis; it was also shown to suppress growth of CTCL cell lines in vitro. Given its low toxicity, enzastaurin has been tested against both solid tumors and hematologic malignancies. This article is focused on the potential role of enzastaurin in the treatment of CTCLs. A phase II multicenter trial evaluated enzastaurin monotherapy in patients with CTCLs. However, the results from this study were disappointing, demonstrating that enzastaurin had only modest clinical activity. Hence, enzastaurin is not currently developed for treating CTCLs. Potential strategies to improve enzastaurin efficacy against CTCLs are discussed: validation of enzastaurin targets such as PKC- $\beta$ expression in CTCL lesions and or/blood; measurement of serum vascular endothelial growth factor levels; dose optimization; combining enzastaurin with other antiangiogenic agents, or glycogen synthase kinase inhibitors, or mammalian target of rapamycin (mTOR) inhibitors. Ultimately, developing more potent inhibitors of PKC- $\beta$ and PI3K/AKT/PTEN/mTOR signaling pathways may be necessary to improve clinical outcomes in CTCLs.

Keywords: cutaneous T-cell lymphomas, enzastaurin, PKC- $\beta$ inhibitors, serine/threonine kinase inhibitors, PI3K/AKT/PTEN pathway

\section{Cutaneous T-cell lymphomas}

Cutaneous T-cell lymphomas (CTCLs) are rare, largely indolent, extranodal nonHodgkin lymphomas characterized by neoplastic T-lymphocyte accumulation in skin. ${ }^{1}$ The two most common types are mycosis fungoides (MF) and the leukemic variant, Sézary syndrome (SS), which account for more than $50 \%$ of all primary cutaneous lymphomas. CTCLs have an age-adjusted incidence rate of 7.7 per 1,000,000 personyears. $^{2}$

The etiology of CTCLs is unclear. It may include genetic predisposition, ${ }^{3,4}$ infectious causes (eg, human T-lymphotropic virus type 1), ${ }^{5,6}$ and environmental factors such as solvents. Malignant T-cells in CTCLs show clonal chromosomal abnormalities, 
mostly deletions and translocations. ${ }^{7-10}$ Increased expression of adhesion molecules such as intercellular adhesion molecule 1, alpha3beta1 integrin, and cutaneous lymphocyte antigen has been observed in CTCLs, which may explain its epidermotropism. ${ }^{11-17}$ Cytokines play an important role in CTCL pathophysiology. Sézary cells, which are present in peripheral blood in SS, show aberrant production of cytokines: increased serum levels of T-helper type 2 cytokines (interleukin [IL]-4, IL-5, and IL-10) and decreased levels of T-helper type 1 cytokines (IL-2 and interferon gamma), which impairs cell-mediated cytotoxicity and increases levels of serum immunoglobulin $\mathrm{E}$ and $\mathrm{A}$, and peripheral eosinophilia.

The categorization of CTCLs as primary cutaneous lymphomas is part of the World Health Organization-European Organisation for Research and Treatment of Cancer classification. ${ }^{18}$ Clinical trials in MF and SS have suffered from a lack of standardization in evaluation, staging, end points, and response criteria. To resolve these inconsistencies, the International Society for Cutaneous Lymphomas, the US Cutaneous Lymphoma Consortium, and the Cutaneous Lymphoma Task Force of the European Organization for Research and Treatment of Cancer developed consensus guidelines. ${ }^{19}$

MF typically affects older adults (median age is $55-60$ years) but has been observed in individuals $\leq 35$. Men are affected more often than women $(2: 1),{ }^{18}$ and blacks are affected more commonly than whites. MF has an indolent course with slow clinical progression (years to decades). Clinical manifestations are varied, from limited cutaneous patches to more extensive, generalized cutaneous plaques (Figure 1) and tumors, commonly accompanied by pruritus. Patches are characteristically located in sun-protected areas, such as the lower abdomen and buttocks. Tumors tend to occur in more advanced disease and are often associated with previous patches or plaques. These lesions frequently become ulcerated, leading to secondary infections and high morbidity. Histology varies with disease stage, but epidermotropism is typically seen; intradermal collections of atypical cells (Pautrier microabscesses) are rare, but when present indicate MF. Immunophenotypic analysis of malignant lymphocytes reveals T-cell markers, typically with a $\mathrm{CD} 4^{+} /$ $\mathrm{CD}^{-}$(T-helper) phenotype, including $\mathrm{CD} 2^{+}, \mathrm{CD}^{+}, \mathrm{CD}^{+}$, $\mathrm{CD}^{+}, \mathrm{CD}^{-} 5 \mathrm{RO}^{+}$, and $\mathrm{CD} 8^{-}$expression. At later stages, patients may exhibit nodal or visceral involvement.

MF staging is based on the TNMB system, which incorporates evaluation of skin tumors $(\mathrm{T})$, lymph nodes $(\mathrm{N})$, viscera (M), and blood (B). Stages IA to IIA represent early

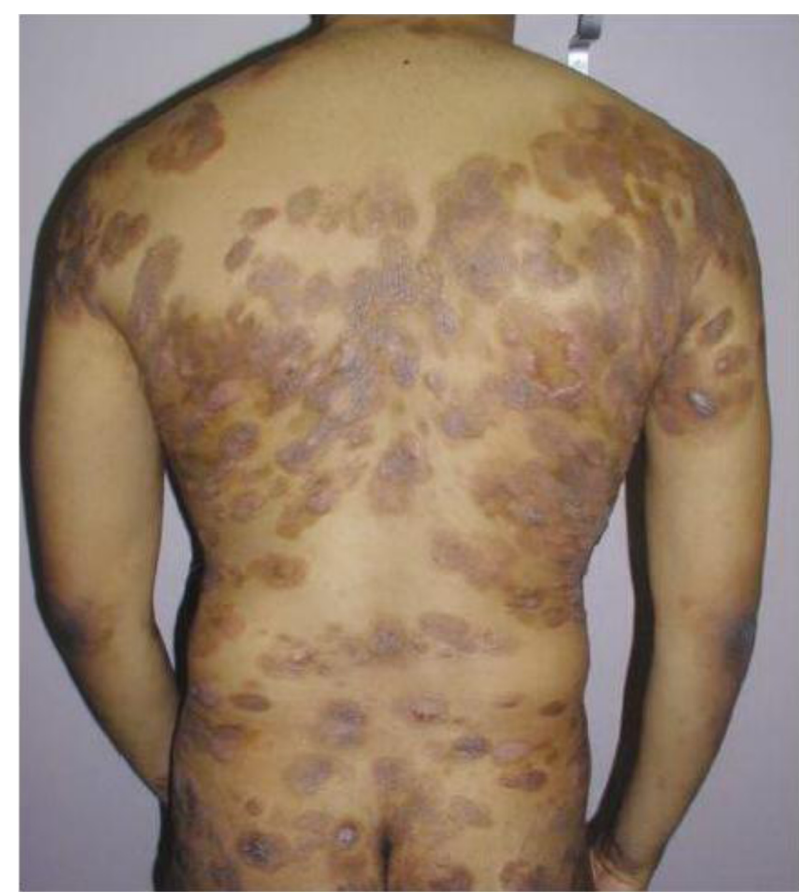

Figure I Mycosis fungoides cutaneous patches involving back and buttocks. (C) 2003. Reproduced with permission of Elsevier. Querfeld C, Guitart J, Kuzel TM, Rosen ST. Primary cutaneous lymphomas: a review with current treatment options. Blood Rev. 2003; | (3): |31-142.75

stage disease; stages IIB to IV and Sézary syndrome represent advanced disease. Prognosis depends on stage, type and extent of cutaneous lesions, and presence of extracutaneous disease. Patients with limited stage MF (disease affecting $\leq 10 \%$ of the skin surface) have a similar life expectancy to an age-, sex-, and race-matched control population, with 10 -year diseasespecific survival of $97 \%-98 \%{ }^{20,21}$ Patients with more extensive disease (higher stage) show decreased overall survival. In a study examining long-term outcomes of $525 \mathrm{MF}$ and SS patients, the most important clinical factors predicting survival were age, $\mathrm{T}$ classification, and extracutaneous disease..$^{22}$

SS is the more aggressive leukemic variant of CTCLs. Patients generally present with erythroderma, pruritus, and characteristic Sézary cells that are circulating mononuclear malignant $\mathrm{T}$ lymphocytes with cerebriform nuclei (Figure 2). In advanced cases, patients suffer from alopecia, leonine facies, hyperkeratosis, cutaneous pain, and ectropion. Prognosis is poor, with a median survival of $2-4$ years. ${ }^{18}$

\section{Currently available treatments for CTCLs}

Several treatment options are available for CTCLs, but there is currently no curative therapy other than allogeneic stem cell transplant. ${ }^{23}$ Treatment is based on disease stage, early (IA-IIA) or advanced (IIB-IVB). In early stage disease, 


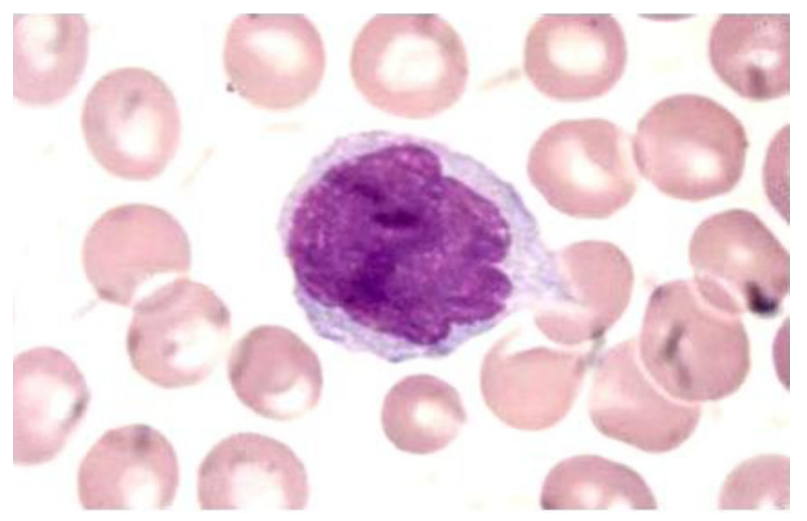

Figure 2 Sézary cells with its characteristic cerebriform nucleus. (C) 2003. Reproduced with permission of Elsevier. Querfeld C, Guitart J, Kuzel TM, Rosen ST. Primary cutaneous lymphomas: a review with current treatment options. Blood Rev. 2003; 17(3):|3|-|42. ${ }^{75}$

skin-directed therapies are preferred: phototherapy with UVB and PUVA, topical chemotherapy (nitrogen mustard or carmustine), topical steroids and retinoids, and irradiation with $\mathrm{X}$-rays or electron beam. In advanced-stage disease, treatment goals include relieving symptoms, reducing tumor burden, and delaying disease progression. Advanced disease is usually treated with a combination of topical and systemic treatments: total skin electron beam radiation, chemotherapy, extracorporeal photopheresis, interferon alpha, newer retinoids such as bexarotene, monoclonal antibodies such as alemtuzumab, and histone deacetylase (HDAC) inhibitors such as vorinostat and romidepsin. It is unclear whether these treatments prolong overall survival in patients with CTCLs. Furthermore, comparative effectiveness of these approaches is unknown because the disease is rare and it is difficult to accrue enough patients for a large randomized phase II or III trial. ${ }^{24}$

Recently, several novel agents were developed for treating CTCLs. One of these agents, pralatrexate, a novel antifolate agent, was approved for unlabeled use in the treatment of relapsed/refractory CTCLs based on the results from a multicenter dose-finding study demonstrating that pralatrexate has high antineoplastic activity with acceptable toxicity. ${ }^{25}$

Bexarotene, a synthetic retinoid $\mathrm{X}$ receptor-selective retinoid agonist, was approved for treating cutaneous manifestations of CTCLs in patients refractory to at least one prior systemic therapy. Approval was based on a phase II-III trial for treatment of advanced-stage (IIB-IVB) CTCLs in 94 patients. ${ }^{26}$ Response rates were $45 \%$ and $55 \%$, but only $6 \%$ achieved a complete response. ${ }^{26}$ Major side effects such as hyperlipidemia and hypothyroidism potentially limit the long-term use of this drug. While retinoids are commonly used in combination with other treatment modalities, including interferon, phototherapy, or radiation, a study comparing bexarotene alone or with the addition of interferon alpha- $2 \mathrm{~b}$ did not find an improved response rate with the use of the combination. ${ }^{27}$

Vorinostat, an oral inhibitor of histone deacetylases (HDAC), was approved for treating CTCL patients with progressive, persistent, or recurrent disease. In a phase IIB multicenter trial of oral vorinostat in 74 patients with stage IB-IVA persistent, progressive, or treatment-refractory MF or SS, the overall response rate was modest at $29.7 \%$, with only one patient achieving a complete response. ${ }^{28}$ Most notably, $32 \%$ of patients had significant relief of pruritus symptoms with vorinostat therapy. The most common drugrelated adverse events included diarrhea, fatigue, and nausea; grade 3 or higher adverse events included fatigue, pulmonary embolism, thrombocytopenia, and nausea. ${ }^{28}$

Depsipeptide (also known as romidepsin) is one of the first HDAC inhibitors found to have activity in CTCL. An international phase II trial evaluated the efficacy of depsipeptide in patients with treatment-refractory CTCLs. ${ }^{29}$ Ninety-six patients with stage IB-IVA CTCLs who had received one or more prior systemic therapies were enrolled. The majority of patients $(71 \%)$ had advanced-stage disease ( $\geq$ IIB). The overall response rate was $34 \%$, with five patients having complete responses. Drug-related toxicities included nausea, thrombocytopenia, leucopenia, and reversible electrocardiographic changes.

Denileukin diftitox is a novel recombinant fusion protein consisting of peptide sequences for the enzymatically active and membrane translocation domains of diphtheria toxin and human IL-2. A phase III study investigated the efficacy, safety and pharmacokinetics of denileukin diftitox in previously treated patients with stage IB-IVA CTCLs that expressed CD25, the receptor for IL-2. ${ }^{30}$ This study demonstrated an overall response rate of $30 \%$, with $10 \%$ of patients obtaining a complete response. Adverse events included flu-like symptoms, acute infusion-related events, vascular leak syndrome, transient elevations in hepatic transaminase levels, and hypoalbuminemia.

Alemtuzumab (Campath-1H), a humanized monoclonal antibody directed against CD52, was effective against CTCLs. ${ }^{31-33}$ In a phase II trial of patients with advanced stage MF or SS, the overall response rate was $55 \%$, with $32 \%$ achieving complete remission. ${ }^{32}$ The median time to treatment failure was 12 months. Alemtuzumab was associated with significant immunosuppression, serious infections (cytomegalovirus, generalized herpes simplex, fatal aspergillosis, fatal Mycobacterium pneumonia), and cardiac toxicity. ${ }^{31-34}$ Low-dose subcutaneous alemtuzumab 
administered for a short time had a good toxicity profile and similar efficacy in SS patients. ${ }^{35}$

Thus, although several biologic agents have recently been FDA approved for the treatment of CTCLs, the average response rate in the frontline setting is only $25 \%-30 \%,{ }^{23}$ which underscores the need for novel agents for these diseases. New agents are currently being investigated for CTCLs. Among them are forodesine, an orally available analog inhibitor of purine nucleoside phosphorylase, and zanolimumab, a fully human monoclonal anti-CD4 antibody. There continue to be additional avenues of investigation into various treatment modalities for CTCL patients. Researchers continue to develop unique agents and novel approaches, such as vaccines, combined modality treatments, and stem cell transplantation. One newer agent investigated in CTCLs is the serine/threonine kinase inhibitor enzastaurin (LY317615; Eli Lilly, Indianapolis, IN).

\section{Enzastaurin}

\section{Structure and mechanism of action}

Enzastaurin is an oral synthetic macrocyclic bisindolylmaleimide. It is a small-molecule serine/threonine kinase inhibitor with specificity for protein kinase $C$ isozyme $\beta$ (PKC- $\beta$ ). $\mathrm{PKC}$ is a receptor for phorbol esters, which are potent carcinogens, capable of inducing tumors in experimental animals. ${ }^{36} \mathrm{PKC}$ is a serine/threonine kinase that becomes activated due to growth factor signaling and translocates to a cell membrane, where it plays a critical role in stimulating cell growth and proliferation (Figure 3). At the membrane, PKC phosphorylates its substrates, resulting in initiation of PI3-kinase (PI3K) signaling and a subsequent cascade of downstream signals, including phosphoinositide-dependent protein kinase 1 , AKT and glycogen synthase kinase (GSK-3) $\beta$. PKC isoforms have been implicated in many aspects of tumorigenesis, including proliferation, differentiation, and apoptosis. Overexpression of the PKC- $\beta_{1}$ isoform results in susceptibility to malignant transformation. ${ }^{36} \mathrm{PKC}$ is an important molecule in PI3K/AKT/phosphatase and tensin homolog (PTEN) signaling (Figure 3), a key pathway regulating cell growth and angiogenesis. ${ }^{37,38}$

Increased levels of PKC have been observed in solid tumors. Mouse models show that inhibition of PKC activity impairs tumor growth. ${ }^{39}$ Overexpression of PKC- $\beta$ has been reported in $\mathrm{B}$-cell and T-cell lymphomas; ${ }^{40,41} \mathrm{PKC}-\beta$ overexpression was associated with inferior prognosis in diffuse large B-cell lymphomas. ${ }^{40}$ Given the role of PKC and specifically PKC- $\beta$ in tumorigenesis, novel agents

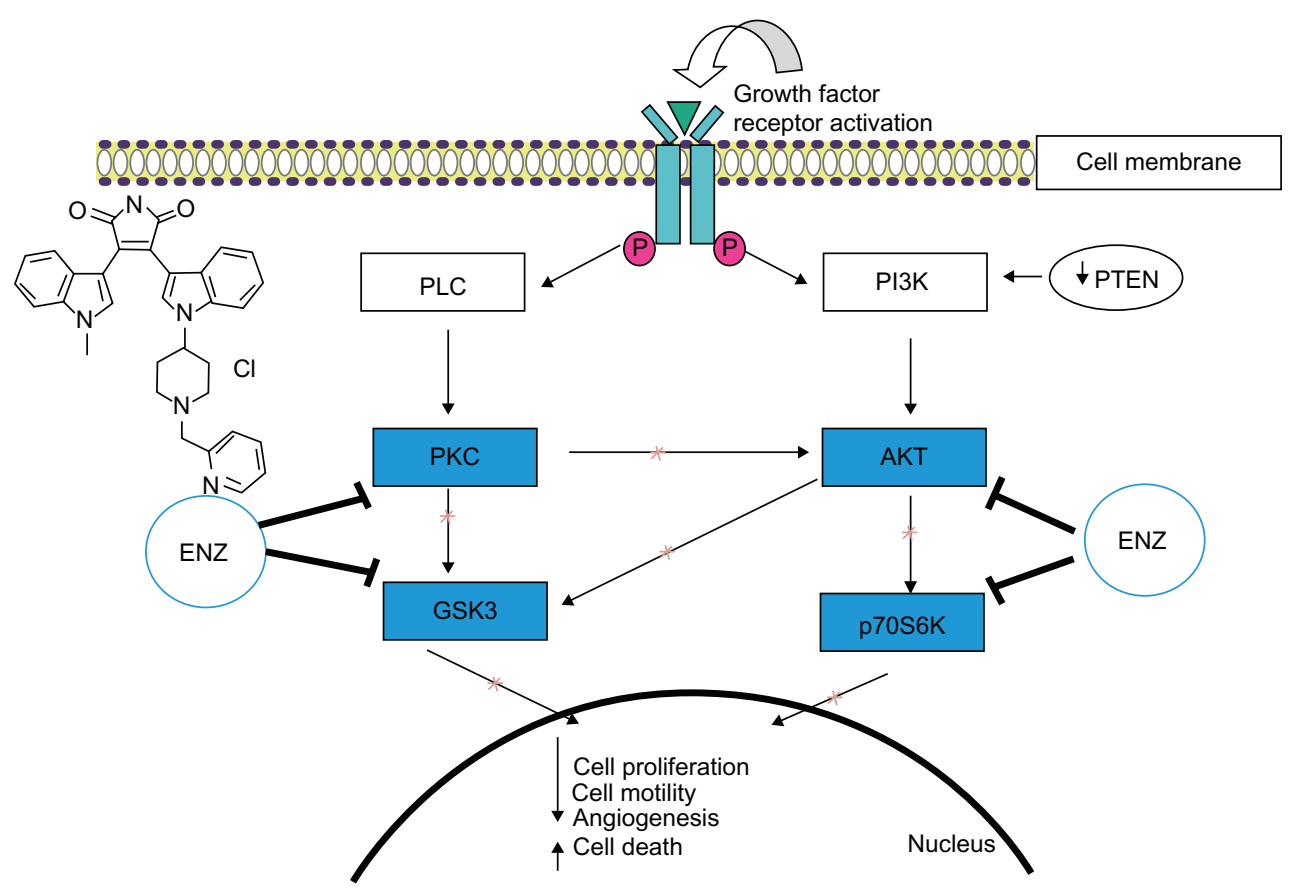

Figure 3 Schematic illustration of the effects of enzastaurin on the PI3K/AKT/PTEN signal transduction pathway.

Notes: In malignant cells, growth factor receptor activation initiates a phosphorylation cascade to activate key signaling proteins. Enzastaurin inhibits protein kinase $C$ activity, phosphorylation, and activation of AKT, GSK3, and S6K. This leads to inhibition of tumor cell proliferation, suppression of tumor-induced angiogenesis, and induction of apoptosis. (c) 2006. Reproduced with permission of American Society of Clinical Oncology. Carducci MA, Musib L, Kies MS, et al. Phase I dose escalation and pharmacokinetic study of enzastaurin, an oral protein kinase $C$ beta inhibitor, in patients with advanced cancer. J Clin Oncol. 2006;24(25):4092-4099. ${ }^{42}$

Abbreviations: ENZ, enzastaurin; PLC, phospholipase C; PKC, protein kinase C; GSK3, glycogen synthase kinase 3; PI3K, phosphoinositide-3 kinase; PDKI, phosphoinositidedependent protein kinase I; AKT, also called protein kinase B (PKB); p70S6K, p70S6 kinase; PTEN, phosphatase and tensin homolog. 
blocking this target may provide promising targeted anticancer therapy.

Enzastaurin was originally developed as an adenosine triphosphatase-competitive selective inhibitor of PKC- $\beta$. It actively competes with adenosine triphosphatase for the binding site of PKC, thereby blocking its activation of PKC- $\beta$ and preventing phosphorylation of downstream molecules. Enzastaurin suppresses the PI3K/AKT/PTEN pathway and inhibits GSK-3 phosphorylation. ${ }^{42}$ Studies with tumor cell lines have shown that the addition of enzastaurin to cell cultures results in inhibition of AKT and GSK-3 $\beta$ phosphorylation. Enzastaurin has both direct and indirect antitumor effects. Enzastaurin inhibition of PKC- $\beta$ and its downstream mediators induces apoptotic cell death and decreased cell proliferation in in vitro experiments. ${ }^{43}$ In vivo experiments show that enzastaurin reduces vascular endothelial growth factor (VEGF) expression and microvessel density in a human xenograft model. ${ }^{44}$ It also suppresses growth of new blood vessels in a rat corneal micropocket assay. ${ }^{45}$ The potent antiangiogenic activity of enzastaurin in preclinical studies stimulated its clinical development.

Enzastaurin is a noncytotoxic agent with a favorable sideeffect profile. A phase I trial in solid-tumor patients indicated that the drug was well tolerated at doses that achieved a biologically active serum concentration. Toxicities included grade 1 chromaturia (red discoloration of the urine), nausea, diarrhea, fatigue, and rare dose-limiting QTc interval prolongation. ${ }^{42}$ In the phase I trial, the enzastaurin dose was escalated up to $700 \mathrm{mg}$ daily and the maximum tolerated dose was not achieved. ${ }^{42}$

\section{Clinical experience with enzastaurin}

Enzastaurin has been evaluated in both hematologic malignancies and solid tumors as either a single agent or in combination with other therapies. Previous studies with enzastaurin as a single agent in solid tumors - in patients with non-smallcell lung cancer, ${ }^{46}$ metastatic colorectal cancer, ${ }^{47}$ locally advanced and metastatic pancreatic cancer, ${ }^{47-49}$ malignant gliomas, ${ }^{50}$ prostate cancer, ${ }^{49}$ breast cancer, ${ }^{47,49}$ ovarian cancer, or primary peritoneal cancer ${ }^{51}-$ did not result in responses as dramatic as its effect in vitro. Investigations of enzastaurin in various hematologic malignancies were more promising. This may be a result of higher selective expression of the $\mathrm{PKC}-\beta$ isoform in hematopoietic cells.

Preclinical studies showed that enzastaurin has activity in multiple myeloma and Waldenström's macroglobulinemia (WM). ${ }^{52,53}$ A multicenter phase II study was conducted to determine whether further study of single-agent enzastaurin is warranted in patients with previously treated WM. ${ }^{54}$ Twenty-nine patients with WM were treated with oral enzastaurin $250 \mathrm{mg}$ twice daily in 28-day cycles. While no patients achieved a complete response, one had a partial response and seven patients had a minor response, for an overall response rate of $27.6 \%$. Only one patient had a grade 3 drug-related adverse event.

Among hematologic malignancies, PKC- $\beta$ has been shown to have the highest level of expression in mantle cell lymphomas, with overexpression in $>90 \%$ of cases. ${ }^{55}$ A phase II study was conducted in patients with relapsed or refractory mantle cell lymphoma. ${ }^{56}$ Sixty patients with relapsed or refractory mantle cell lymphoma received oral enzastaurin $500 \mathrm{mg}$ daily; the primary end point was freedom from progression (FFP) after three 28-day cycles of treatment. Following three cycles of treatment, 22 of 60 patients $(37 \%)$ attained FFP for $\geq 3$ cycles; six of 22 attained FFP $>6$ months. At the time of publication, ${ }^{56}$ two patients remained on treatment and experienced FFP for $>23$ months. However, no objective tumor responses were noted. In this study, enzastaurin was well tolerated, with fatigue being the most common toxicity. While there were no drug-related deaths, grade 3 anemia, diarrhea, dyspnea, vomiting, hypotension, and syncope were observed.

A phase II study investigated enzastaurin in 55 patients with relapsed or refractory diffuse large B-cell lymphoma. ${ }^{57}$ Enzastaurin was given once daily, initially at $525 \mathrm{mg}$ and subsequently at $500 \mathrm{mg}$, until the disease progressed or unacceptable toxicity occurred. Study end points included FFP for two or more 28-day cycles, an objective response, and toxicity. Overall, twelve of 55 patients $(22 \%)$ experienced FFP for $\geq 2$ cycles, with eight attaining FFP for $\geq 4$ cycles. Four patients, including three complete responders and one patient with stable disease, maintained FFP for 20-50 months. ${ }^{57}$

Enzastaurin was also investigated in a phase II study of 66 patients with grade 1 or 2 stage III or IV follicular lymphoma. ${ }^{58}$ Patients received enzastaurin $500 \mathrm{mg}$ for up to 3 years or until progression, withdrawal, or unacceptable toxicity occurred. The primary end point was response rate; secondary end points included duration of response and safety. At the time of publication, the median overall exposure was 10.1 months. Of the 64 patients who received at least one dose of enzastaurin, one had a complete response and 15 had a partial response, resulting in an overall response rate of $25 \% ; 25(39.1 \%)$ had disease progression. While there were no drug-related deaths, three patients had serious drug-related 


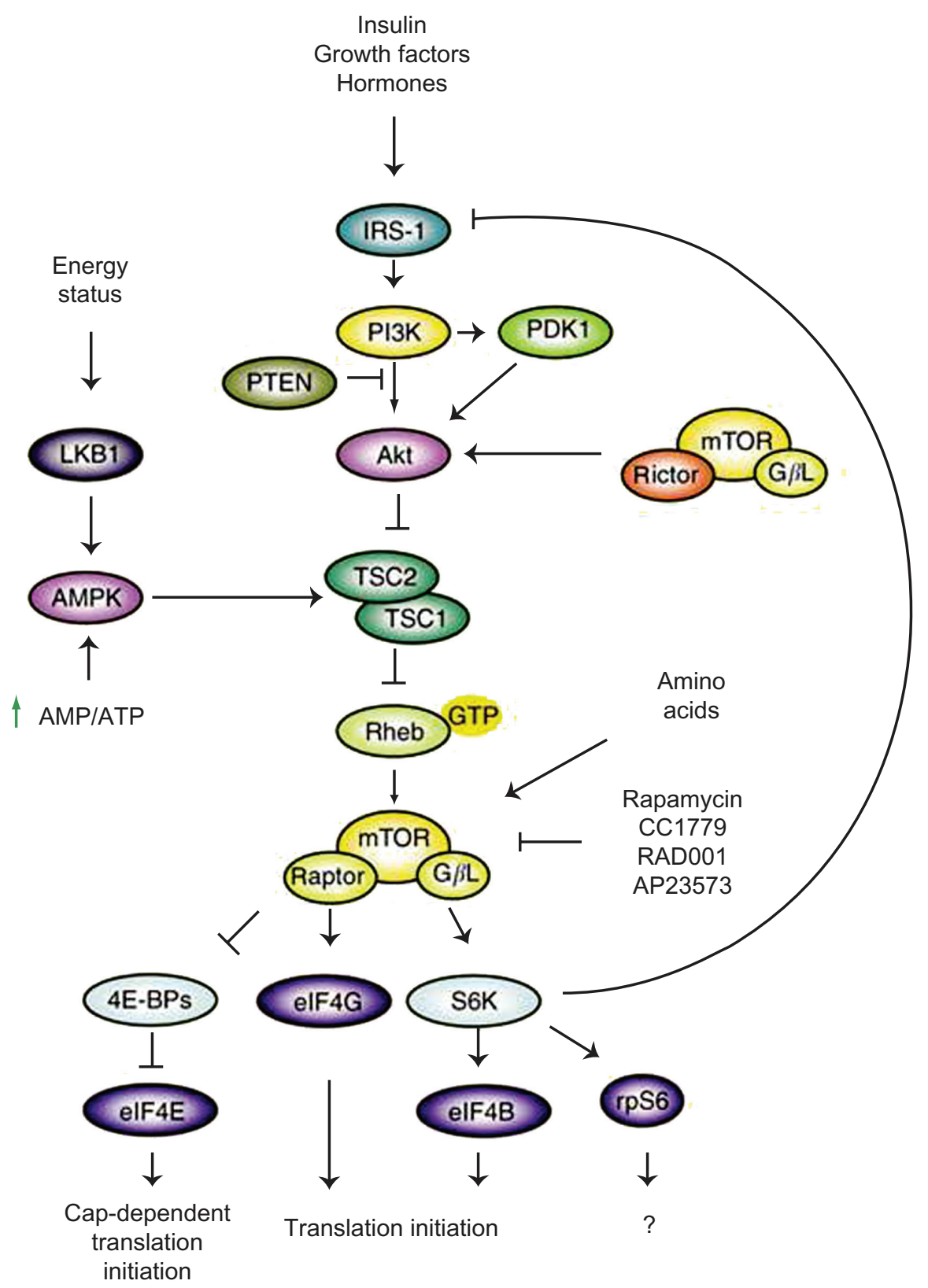

Figure 4 Schematic of the mTOR pathway.

Notes: mTOR receives input from a number of upstream pathways, including PI3K/Akt, TSCI/TSC2, and AMPK, and acts through downstream effectors S6K and E4BPI to exert its effects.

(c) 2007. Reproduced with permission of Nature Publishing Group Hartford CM. Ratain MJ. Rapamycin: something old, something new, sometimes borrowed and now renewed. Clin Pharmacol Ther. 2007;82(4):381-388. ${ }^{76}$

Abbreviations: IRS-I, insulin receptor serine kinase I; PI3K, phosphoinositide-3 kinase; PDKI, phosphoinositide-dependent protein kinase I; PTEN, phosphatase and tensin homolog deleted on chromosome ten; AKT, also called protein kinase B (PKB); mTOR, mammalian target of rapamycin rictor, raptor, G/L - proteins that form complexes with mTOR; LKBI, serine/threonine protein kinase I; AMPK, AMP-activated kinase; TSC2, tuberous sclerosis complex 2; TSCl, tuberous sclerosis complex I; Rheb - GTP-binding protein; CCI779, temsirolimus (an mTOR inhibitor); RAD00I, everolimus (an mTOR inhibitor); AP23573, deferolimus (an mTOR inhibitor); 4E-BPs, EIF4G, S6K - effectors of mTOR; EIF4E, EIF4G, elF4B, mammalian transcription factors; rpS6, ribosomal protein S6 kinase.

adverse events, including grade 3 bronchitis, grade 2 diarrhea, and grade 2 pancreatitis.

\section{Evaluation of enzastaurin in CTCLs}

Despite many therapeutic developments, CTCLs still remain poorly understood and largely incurable. Therefore, novel drugs that target cell proliferation and angiogenesis, including the targeting of PKC- $\beta$ and AKT signaling pathways, are of great interest in the treatment of CTCLs.

Based on these considerations, enzastaurin, an inhibitor of PKC- $\beta$ and the PI3K/AKT/PTEN signaling pathway, was tested in the treatment of CTCLs, which appeared to be dependent on this pathway for survival. In vitro studies demonstrated that enzastaurin has substantial activity against 
two well-characterized human CTCL cell lines, HuT-78 and HH. ${ }^{59}$ Enzastaurin effectively inhibited growth and induced apoptosis in these cell lines through inhibition of AKT signaling in a time- and dose-dependent manner.

A multicenter, open-label screening study was initiated to evaluate the activity of enzastaurin in relapsed peripheral T-cell lymphomas, CTCLs, and indolent and aggressive B-cell lymphomas. Patients received $250 \mathrm{mg}$ oral enzastaurin twice daily in 28-day cycles for up to 2 years and were assessed for overall tumor response, progression-free survival, time to progression, and safety. A total of 57 patients were enrolled, including eleven with CTCLs. Nobody had a complete response; however, partial responses were observed in four of the 57 patients, including one patient with CTCL. Three patients $(5 \%)$ discontinued treatment due to drugrelated toxicities; there were no drug-related deaths. Based on these results, the authors postulated that enzastaurin appeared to have activity and was tolerated in patients with indolent lymphomas, including CTCLs. ${ }^{60}$

Given these promising results, a phase II multicenter trial with a two-stage design was conducted to evaluate enzastaurin monotherapy in patients with heavily pretreated, recurrent, or refractory MF, stage IB-IVB, or SS. However, results from this study were disappointing. Enzastaurin had only modest clinical activity in this patient population. ${ }^{61}$ Twenty-five patients received enzastaurin $250 \mathrm{mg}$ twice daily until disease progression or intolerable toxicity. The primary end point was investigator-assessed response rate, with secondary end points including time to objective response, response duration, time to progression, patient-reported pruritus, and safety/tolerability. A partial response was observed in one patient with MF, with no patient achieving a complete response. The median time to progression was 78 days among patients with MF, and 44 days among patients with SS. Six patients achieved symptomatic relief from pruritus. While enzastaurin was generally well tolerated, with mostly grade 1-2 side effects including fatigue and diarrhea, there were two adverse events with subsequent drug discontinuation after one of these events, desquamation, which possibly was related to treatment. This trial did not proceed to stage 2 of accrual and was terminated because of the insufficient activity of enzastaurin. To our knowledge, no translational, pharmacokinetic, or pharmacodynamic studies were conducted in this trial.

The authors of this study postulated that the lack of meaningful clinical response suggests that another pathway could be involved in the growth and survival of malignant T-lymphocytes. Therefore, monotherapy with a targeted agent such as enzastaurin may not be an effective therapy. The authors also suggested that prolonged exposure may be necessary to reach a therapeutic level of enzastaurin, which may not be appropriate for highly symptomatic CTCL patients.

In summary, clinical trials examining the role of enzastaurin in various malignancies have been negative so far, and enzastaurin is not FDA approved for any clinical indication. The only remaining ongoing clinical trial of enzastaurin is a phase III PRELUDE trial investigating enzastaurin as compared to placebo in the maintenance setting in patients with large-cell lymphomas. The future development of enzastaurin is uncertain at this time. ${ }^{62}$

\section{Discussion and future directions}

Intensive research over the last two decades into the molecular mechanisms of cancer has resulted in a better understanding of the crucial role that specific molecular abnormalities (targets) in signal transduction pathways play in tumor initiation and progression. Several very successful targeted therapies, which block the targets and corresponding upregulated signaling pathways, have been developed for solid and hematologic malignancies such as chronic myeloid leukemia, gastrointestinal stromal tumors, and breast cancer. As research advanced, it became clear that effective targeted therapies require identification of the target that is critical for tumor survival, demonstration of the drug binding to the target, and correlation of the subsequent downstream effect with a clinical outcome. Since there is often "cross-talk" between different and sometimes redundant signal transduction pathways,${ }^{36}$ clinical trials may utilize a combination of agents that block up- and downstream targets in the same signal transduction pathway, or block several signal transduction pathways.

Multiplicity of molecular networks in malignant cells can explain why the disappointing results from enzastaurin clinical trials are not unique. Many targeted agents failed to demonstrate sufficient activity to proceed to stage 2 of accrual in phase II trials or demonstrate enough benefit in phase III trials to obtain FDA approval. Recently, the National Cancer Institute mandated investigators to incorporate tissue assays for validated targets, and demonstrate drug-target interaction as a prerequisite condition in order to obtain federal funding for clinical trials. If the clinical trial fails to document the specific target and enroll patients with the target present in the tumor, it may result in falsely negative data and consequent wasting of valuable resources, especially in rare diseases such as CTCLs. 
Table I Summary of clinical trials with new agents in cutaneous T-cell lymphomas

\begin{tabular}{|c|c|c|c|c|}
\hline Drug & Study objective & Patient population & Response & $\begin{array}{l}\text { Significant adverse } \\
\text { events }\end{array}$ \\
\hline Pralatrexate ${ }^{25}$ & $\begin{array}{l}\text { Dose-finding study } \\
\text { of pralatrexate }\end{array}$ & $\begin{array}{l}\text { Mycosis fungoides, Sézary } \\
\text { syndrome, or primary cutaneous } \\
\text { anaplastic large-cell lymphoma, } \\
\text { with disease progression after } \\
\text { at least I prior systemic therapy }\end{array}$ & $\begin{array}{l}\text { - Overall response was } 43 \% \\
\text { at a dose of } 15 \mathrm{mg} / \mathrm{m}^{2} \\
\text { - Overall response was } 50 \% \\
\text { at a dose of } \geq 15 \mathrm{mg} / \mathrm{m}^{2}\end{array}$ & $\begin{array}{l}\text { Mucositis, } \\
\text { neutropenia, anemia, } \\
\text { thrombocytopenia }\end{array}$ \\
\hline Bexarotene ${ }^{26}$ & $\begin{array}{l}\text { Evaluate safety and } \\
\text { efficacy of } \\
\text { bexarotene }\end{array}$ & $\begin{array}{l}\text { Refractory advanced stage } \\
\text { CTCL (stage IIB-IVB) }\end{array}$ & $\begin{array}{l}\text { - Response rate was } 45 \% \text { at } \\
300 \mathrm{mg} / \mathrm{m}^{2} / \text { day dosing } \\
\text { - Response rate was } \\
55 \% \text { at }>300 \mathrm{mg} / \mathrm{m}^{2} / \text { day dosing } \\
\text { - Only } 6 \% \text { achieved complete } \\
\text { response }\end{array}$ & $\begin{array}{l}\text { Hyperlipidemia, } \\
\text { hypothyroidism }\end{array}$ \\
\hline Vorinostat ${ }^{28}$ & $\begin{array}{l}\text { Evaluate the safety } \\
\text { and activity of } \\
\text { vorinostat }\end{array}$ & $\begin{array}{l}\text { Persistent, progressive, or } \\
\text { treatment refractory mycosis } \\
\text { fungoides or Sézary syndrome } \\
\text { (stage IB-IVA) }\end{array}$ & $\begin{array}{l}\text { - Overall response rate was } 29.7 \% \\
\text { - One patient achieved } \\
\text { a complete response }\end{array}$ & $\begin{array}{l}\text { - Diarrhea, fatigue, nausea, } \\
\text { pulmonary embolism, } \\
\text { thrombocytopenia }\end{array}$ \\
\hline Romidepsin ${ }^{29}$ & $\begin{array}{l}\text { Confirm the } \\
\text { efficacy } \\
\text { of romidepsin }\end{array}$ & $\begin{array}{l}\text { Stage IB-IVA CTCL who } \\
\text { had received one or more } \\
\text { prior systemic therapies }\end{array}$ & $\begin{array}{l}\text { - Overall response rate was } 34 \% \\
\text { - Five patients achieved } \\
\text { a complete response }\end{array}$ & $\begin{array}{l}\text { - Nausea, } \\
\text { thrombocytopenia, } \\
\text { leukopenia, reversible } \\
\text { EKG changes }\end{array}$ \\
\hline $\begin{array}{l}\text { Denileukin } \\
\text { diftitox }{ }^{30}\end{array}$ & $\begin{array}{l}\text { Investigated the } \\
\text { efficacy, safety, and } \\
\text { pharmacokinetics } \\
\text { of denileukin } \\
\text { diftitox }\end{array}$ & $\begin{array}{l}\text { Previously treated patients } \\
\text { with stage IB-IVA CTCL }\end{array}$ & $\begin{array}{l}\text { - Overall response rate was } 30 \% \\
\text { - } 10 \% \text { of patients obtained } \\
\text { a complete response }\end{array}$ & $\begin{array}{l}\text { Flu-like symptoms, acute } \\
\text { infusion-related events, } \\
\text { vascular leak syndrome, } \\
\text { transient elevations in } \\
\text { hepatic transaminase } \\
\text { levels, hypoalbuminemia }\end{array}$ \\
\hline Alemtuzumab ${ }^{32}$ & $\begin{array}{l}\text { Evaluate the safety } \\
\text { and efficacy of } \\
\text { alemtuzumab }\end{array}$ & $\begin{array}{l}\text { Advanced stage mycosis } \\
\text { fungoides or Sézary syndrome } \\
\text { (stage II-IV) }\end{array}$ & $\begin{array}{l}\text { - Overall response rate was } 55 \% \\
\text { - } 32 \% \text { achieved a complete } \\
\text { remission }\end{array}$ & $\begin{array}{l}\text { Significant } \\
\text { immunosuppression } \\
\text { and serious infections, } \\
\text { cardiac toxicity }\end{array}$ \\
\hline
\end{tabular}

Abbreviations: CTCL, cutaneous T-cell lymphoma; EKG, electrocardiogram.

With these considerations in mind, further development of enzastaurin in CTCLs is likely to depend on target validation. It is thus imperative to study overexpression of the PKC- $\beta$ isoform in CTCL cell lines and clinical samples. Murine xenograft models of CTCLs have been established with subcutaneous injection of Sézary cells or malignant T-lymphocytes in immunodeficient mice. ${ }^{63,64}$ These models recapture, albeit incompletely, the course of CTCLs and can be instrumental in confirming PKC- $\beta$ overexpression in CTCLs.

It is also possible that we have not yet found a reliable biomarker of enzastaurin activity. Anecdotal cases of dramatic and durable responses have been reported in clinical trials with enzastaurin, but these findings could not have been predicted or explained with the existing battery of translational biomarkers. We have personally observed a patient with recurrent ovarian cancer who has been progression-free for 5 years on enzastaurin. Extensive tumor and blood testing for mutations and gene copy numbers as well as protein expression of PKC- $\beta$, pGSK3, PTEN, TP53, PIK3CA, AKT2, and other relevant biomarkers of the PI3K/AKT/PTEN pathway failed to explain this dramatic response..$^{51}$ No correlations between biomarkers and clinical outcomes have been noted in other clinical trials with enzastaurin. ${ }^{65}$ The results of the aforementioned PRELUDE trial are expected to become available in 2012. This study investigated a panel of biomarkers implicated in enzastaurin's mechanism, including PKC- $\beta$, pGSK3, and others. Hopefully, some of these biomarkers will correlate with clinical outcomes in the trial. This would allow revisiting the role of enzastaurin in CTLCs by selecting patients with the appropriate biomarkers. If, however, no reliable biomarker is identified in the PRELUDE trial, the next step may be global assessment of genetic changes in CTCL lesions, and/or peripheral blood using commercial genomic assays or next-generation sequencing.

As mentioned earlier, enzastaurin has antiangiogenic properties. Thus, another potential biomarker for selecting CTCL patients for a clinical trial with enzastaurin is a biomarker of angiogenic activity either in the tumor or in the host. Identification and validation of angiogenic markers has 
been the holy grail of the development of bevacizumab and of other antiangiogenic drugs. Various markers have been investigated for this purpose, including microvessel density in tumors and single-nucleotide polymorphisms and other aberrations in the $V E G F$ gene. ${ }^{66}$ Although not confirmed by all studies, the baseline serum level of VEGF has emerged as the best biomarker for predicting which patients are more likely to respond to antiangiogenic interventions (patients with a high VEGF level are more likely to respond). ${ }^{67}$ Given this information, future clinical trials with enzastaurin in CTCLs may benefit from selecting patients with high serum VEGF levels or a more reliable biomarker of tumor angiogenic activity if one is found.

Because CTCLs involve primarily skin, access to tumor tissue is relatively easy compared to other malignancies. Once a target is confirmed, that information should guide the design of subsequent enzastaurin clinical trials. Selection of study participants should include patients whose tumor biopsies and/or serum specimens are available for testing and are shown to express the biomarker (high levels of the PKC- $\beta$ isoform or of VEGF). Although this strategy is appealing, limiting the patient population may impede the development of enzastaurin in such a rare disease as CTCLs and necessitate an international trial to obtain sufficient statistical power and arrive at a meaningful conclusion.

Since biologic agents are usually much less toxic compared to chemotherapeutic drugs, it is often difficult to find the optimal dose. As discussed earlier, the MTD was not achieved in the phase I trial with enzastaurin doses up to $700 \mathrm{mg} /$ day. ${ }^{42}$ This is a common experience in phase I clinical trials with targeted agents. Hence, dose escalation and achieving higher serum concentrations of enzastaurin may be necessary to achieve a more potent biologic effect. For enzastaurin, a dose of $525 \mathrm{mg} /$ day was recommended for subsequent phase II trials with a goal for the serum concentration of the drug being $1400 \mathrm{nmol} / \mathrm{L}$. It has been suggested that the optimal biologic dose of enzastaurin should be based on the serum concentration of the drug that inhibits $90 \%\left(\mathrm{IC}_{90}\right)$ of PKC- $\beta$ activity ${ }^{68}$ rather than on the absolute free drug concentration. The activity of PKC decreases in monocytes in peripheral blood samples after exposure to enzastaurin ${ }^{69}$ and may assist in establishing the optimal biologic dose of enzastaurin.

Multiple clinical trials with various targeted agents often demonstrate a low level of single-agent activity. A solution to this problem may lie in studying combinations of the biologic agent with chemotherapeutic agents or other targeted drugs. Combinations of enzastaurin with other active agents may be beneficial in the treatment of CTCLs. It is reasonable to combine enzastaurin with other agents inhibiting downstream molecules in the PI3K/AKT/PTEN pathway to block the pathway more effectively. This approach has been tried in vitro with a combination of enzastaurin and the phosporylated glycogen synthase kinase inhibitor AR-A014418. Enhanced cytotoxicity was observed when CTCL cell lines HH, H9, and MJ were exposed to this combination. The authors suggest further in vivo experiments in mice to investigate possible synergistic toxicities of this combination. ${ }^{70}$

As discussed earlier, signal transduction pathways can be redundant and overlapping. For instance, the PI3K/AKT/ PTEN signal transduction pathway and the mTOR pathway interact and are complementary.

Recently, a number of mTOR inhibitors entered clinical trials and showed promise in various tumor types. One of them, everolimus, has been FDA approved for renal cell carcinoma and has been studied in combinations with other cytostatic agents with encouraging results. ${ }^{71}$ Given this data, it is reasonable to study everolimus as a single agent and in combination with enzastaurin in vitro in CTCLs.

Another potential strategy for developing enzastaurinbased combinations in CTCLs is to administer it with agents affecting the same biologic processes ("hallmarks of cancer" ${ }^{\prime 2}$ ): angiogenesis and apoptosis. This approach has been tried with a combination of enzastaurin and another antiangiogenic agent - bevacizumab. This combination showed a dramatic response and only minimally increased toxicity in heavily pretreated women with gynecologic cancers, with $74 \%$ of patients achieving clinical benefit. ${ }^{73}$

Unfortunately, the development of drug combinations for the treatment of malignant diseases is hampered by the FDA requirement for a relatively high level of single-agent activity as well as the difficulty of the licensing processes for agents patented by different pharmaceutical companies. Therefore, combination therapies from the same company are those that are most likely to succeed. Since Eli Lilly currently holds the license for enzastaurin, it has the ability to study enzastaurin in combination with mTOR inhibitors or antiangiogenic agents either currently available or under development, with the hope of finding a powerful regimen for the treatment of CTCLs.

Enzaustarin can potentially be combined with already approved agents for CTCLs, such as HDAC inhibitors ${ }^{74}$ and interferon alpha. ${ }^{61}$ However, to demonstrate superiority, such a trial would require minimal overlapping toxicities of two agents and a large number of patients to conduct a randomized phase II trial, which may not be feasible in CTCLs. 
Future development of new therapies for the treatment of CTCLs may abandon enzastaurin altogether, but capitalize on the lessons learned in enzastaurin trials. Novel therapeutics that can more potently inhibit PKC- $\beta$ and the PI3K/AKT/PTEN/mTOR signaling pathway are currently under investigation, and may eventually change the clinical outcome of CTCLs. Since developing new agents for the treatment of a rare disease is such a challenge, it is crucial for industry, government, philanthropy, and patientadvocacy organizations to work together to prolong lives and achieve a cure.

\section{Acknowledgments}

The authors are very grateful to Parameswaran Venugopal, $\mathrm{MD}$, for his thoughtful review of the manuscript and to Jeremy Fields, PhD, and Jill McHugh for the valuable assistance in manuscript preparation.

\section{Disclosure}

The authors report no conflicts of interest in this work.

\section{References}

1. Siegel RS, Pandolfino T, Guitart J, Rosen S, Kuzel TM. Primary cutaneous T-cell lymphoma: review and current concepts. J Clin Oncol. 2000;18(15):2908-2925.

2. Bradford PT, Devesa SS, Anderson WF, Toro JR. Cutaneous lymphoma incidence patterns in the United States: a population-based study of 3884 cases. Blood. 2009;113(21):5064-5073.

3. van Doorn R, Zoutman WH, Dijkman R, et al. Epigenetic profiling of cutaneous T-cell lymphoma: promoter hypermethylation of multiple tumor suppressor genes including BCL7a, PTPRG, and p73. J Clin Oncol. 2005;23(17):3886-3896.

4. Shin J, Monti S, Aires DJ, et al. Lesional gene expression profiling in cutaneous T-cell lymphoma reveals natural clusters associated with disease outcome. Blood. 2007;110(8):3015-3027.

5. Hall WW, Liu CR, Schneewind O, et al. Deleted HTLV-I provirus in blood and cutaneous lesions of patients with mycosis fungoides. Science. 1991;253(5017):317-320.

6. Ghosh SK, Abrams JT, Terunuma H, Vonderheid EC, DeFreitas E. Human T-cell leukemia virus type I tax/rex DNA and RNA in cutaneous T-cell lymphoma. Blood. 1994;84(8):2663-2671.

7. Johnson GA, Dewald GW, Strand WR, Winkelmann RK. Chromosome studies in 17 patients with the Sézary syndrome. Cancer. 1985;55(10):2426-2433.

8. Karenko L, Hahtola S, Paivinen S, et al. Primary cutaneous T-cell lymphomas show a deletion or translocation affecting NAV3, the human UNC-53 homologue. Cancer Res. 2005;65(18):8101-8110.

9. Thangavelu M, Finn WG, Yelavarthi KK, et al. Recurring structural chromosome abnormalities in peripheral blood lymphocytes of patients with mycosis fungoides/Sézary syndrome. Blood. 1997;89(9): 3371-3377.

10. Karenko L, Hyytinen E, Sarna S, Ranki A. Chromosomal abnormalities in cutaneous T-cell lymphoma and in its premalignant conditions as detected by G-banding and interphase cytogenetic methods. $J$ Invest Dermatol. 1997;108(1):22-29.

11. Shiohara T, Moriya N, Gotoh C, et al. Differential expression of lymphocyte function-associated antigen 1 (LFA-1) on epidermotropic and non-epidermotropic T-cell clones. J Invest Dermatol. 1989;93(6): 804-808.
12. Nickoloff BJ, Griffiths CE, Baadsgaard O, Voorhees JJ, Hanson CA, Cooper KD. Markedly diminished epidermal keratinocyte expression of intercellular adhesion molecule-1 (ICAM-1) in Sézary syndrome. JAMA. 1989;261(15):2217-2221.

13. Savoia P, Novelli M, Fierro MT, Cremona O, Marchisio PC, Bernengo MG. Expression and role of integrin receptors in Sézary syndrome. J Invest Dermatol. 1992;99(2):151-159.

14. Heald PW, Yan SL, Edelson RL, Tigelaar R, Picker LJ. Skin-selective lymphocyte homing mechanisms in the pathogenesis of leukemic cutaneous T-cell lymphoma. J Invest Dermatol. 1993;101(2):222-226.

15. Borowitz MJ, Weidner A, OlsenEA, Picker LJ.Abnormalities of circulating T-cell subpopulations in patients with cutaneous T-cell lymphoma: cutaneous lymphocyte-associated antigen expression on T cells correlates with extent of disease. Leukemia. 1993;7(6):859-863.

16. Girardi M, Heald PW, Wilson LD. The pathogenesis of mycosis fungoides. N Engl J Med. 2004;350(19):1978-1988.

17. Lopez-Lerma I, Estrach MT. A distinct profile of serum levels of soluble intercellular adhesion molecule-1 and intercellular adhesion molecule-3 in mycosis fungoides and Sézary syndrome. $J$ Am Acad Dermatol. 2009;61(2):263-270.

18. Willemze R, Jaffe ES, Burg G, et al. WHO-EORTC classification for cutaneous lymphomas. Blood. 2005;105(10):3768-3785.

19. Olsen EA, Whittaker S, Kim YH, et al. Clinical end points and response criteria in mycosis fungoides and Sézary syndrome: a consensus statement of the International Society for Cutaneous Lymphomas, the United States Cutaneous Lymphoma Consortium, and the Cutaneous Lymphoma Task Force of the European Organization for Research and Treatment of Cancer. J Clin Oncol. 2011;29(18):2598-2607.

20. Kim YH, Jensen RA, Watanabe GL, Varghese A, Hoppe RT. Clinical stage IA (limited patch and plaque) mycosis fungoides. A long-term outcome analysis. Arch Dermatol. 1996;132(11):1309-1313.

21. Kim YH, Chow S, Varghese A, Hoppe RT. Clinical characteristics and long-term outcome of patients with generalized patch and/or plaque (T2) mycosis fungoides. Arch Dermatol. 1999;135(1):26-32.

22. Kim YH, Liu HL, Mraz-Gernhard S, Varghese A, Hoppe RT. Long-term outcome of 525 patients with mycosis fungoides and Sézary syndrome: clinical prognostic factors and risk for disease progression. Arch Dermatol. 2003;139(7):857-866.

23. Lansigan F, Foss FM. Current and emerging treatment strategies for cutaneous T-cell lymphoma. Drugs. 2010;70(3):273-286.

24. Hughes DA, Tunnage B, Yeo ST. Drugs for exceptionally rare diseases: do they deserve special status for funding? QJM. 2005;98(11):829-836.

25. Horwitz SM, Kim YH, Foss FM, et al. Identification of an active, well-tolerated dose of pralatrexate in patients with relapsed or refractory cutaneous T-cell lymphoma (CTCL): final results of a multicenter dose-finding study. ASH Annu Meet Abstr. 2010;116(21):2800.

26. Duvic M, Hymes K, Heald P, et al. Bexarotene is effective and safe for treatment of refractory advanced-stage cutaneous T-cell lymphoma: multinational phase II-III trial results. J Clin Oncol. 2001;19(9):2456-2471.

27. Straus DJ, Duvic M, Kuzel T, et al. Results of a phase II trial of oral bexarotene (targretin) combined with interferon alfa- $2 \mathrm{~b}$ (intron-A) for patients with cutaneous T-cell lymphoma. Cancer. 2007;109(9):1799-1803.

28. Olsen EA, Kim YH, Kuzel TM, et al. Phase IIb multicenter trial of vorinostat in patients with persistent, progressive, or treatment refractory cutaneous T-cell lymphoma. J Clin Oncol. 2007;25(21):3109-3115.

29. Whittaker SJ, Demierre MF, Kim EJ, et al. Final results from a multicenter, international, pivotal study of romidepsin in refractory cutaneous T-cell lymphoma. J Clin Oncol. 2010;28(29):4485-4491.

30. Olsen E, Duvic M, Frankel A, et al. Pivotal phase III trial of two dose levels of denileukin diftitox for the treatment of cutaneous T-cell lymphoma. J Clin Oncol. 2001;19(2):376-388.

31. Enblad G, Hagberg H, Erlanson M, et al. A pilot study of alemtuzumab (anti-CD52 monoclonal antibody) therapy for patients with relapsed or chemotherapy-refractory peripheral T-cell lymphomas. Blood. 2004;103(8):2920-2924. 
32. Lundin J, Hagberg H, Repp R, et al. Phase 2 study of alemtuzumab (anti-CD52 monoclonal antibody) in patients with advanced mycosis fungoides/Sézary syndrome. Blood. 2003;101(11):4267-4272.

33. Kennedy GA, Seymour JF, Wolf M, et al. Treatment of patients with advanced mycosis fungoides and Sézary syndrome with alemtuzumab. Eur J Haematol. 2003;71(4):250-256.

34. Lenihan DJ, Alencar AJ, Yang D, Kurzrock R, Keating MJ, Duvic M. Cardiac toxicity of alemtuzumab in patients with mycosis fungoides/ Sézary syndrome. Blood. 2004;104(3):655-658.

35. Bernengo MG, Quaglino P, Comessatti A, et al. Low-dose intermittent alemtuzumab in the treatment of Sézary syndrome: clinical and immunologic findings in 14 patients. Haematologica. 2007;92(6):784-794.

36. Weinstein IB. Nonmutagenic mechanisms in carcinogenesis: role of protein kinase $\mathrm{C}$ in signal transduction and growth control. Environ Health Perspect. 1991;93:175-179.

37. Jiang BH, Liu LZ. PI3K/PTEN signaling in angiogenesis and tumorigenesis. Adv Cancer Res. 2009;102:19-65.

38. Hafsi S, Pezzino FM, Candido S, et al. Gene alterations in the $\mathrm{PI} 3 \mathrm{~K} / \mathrm{PTEN} / \mathrm{AKT}$ pathway as a mechanism of drug-resistance [review] Int J Oncol. 2012;40(3):639-644.

39. Dean N, McKay R, Miraglia L, et al. Inhibition of growth of human tumor cell lines in nude mice by an antisense of oligonucleotide inhibitor of protein kinase C-alpha expression. Cancer Res. 1996;56(15):3499-3507.

40. Hans CP, Weisenburger DD, Greiner TC, et al. Expression of PKCbeta or cyclin D2 predicts for inferior survival in diffuse large B-cell lymphoma. Mod Pathol. 2005;18(10):1377-1384.

41. Gorelik G, Barreiro Arcos ML, Klecha AJ, Cremaschi GA. Differential expression of protein kinase $\mathrm{C}$ isoenzymes related to high nitric oxide synthase activity in a T lymphoma cell line. Biochim Biophys Acta. 2002;1588(2):179-188.

42. Carducci MA, Musib L, Kies MS, et al. Phase I dose escalation and pharmacokinetic study of enzastaurin, an oral protein kinase $\mathrm{C}$ beta inhibitor, in patients with advanced cancer. J Clin Oncol. 2006;24(25):4092-4099.

43. Graff JR, McNulty AM, Hanna KR, et al. The protein kinase cbetaselective inhibitor, enzastaurin (LY317615.HCl), suppresses signaling through the AKT pathway, induces apoptosis, and suppresses growth of human colon cancer and glioblastoma xenografts. Cancer Res 2005;65(16):7462-7469.

44. Keyes KA, Mann L, Sherman M, et al. LY317615 decreases plasma VEGF levels in human tumor xenograft-bearing mice. Cancer Chemother Pharmacol. 2004;53(2):133-140.

45. Teicher BA, Alvarez E, Menon K, et al. Antiangiogenic effects of a protein kinase cbeta-selective small molecule. Cancer Chemother Pharmacol. 2002;49(1):69-77.

46. Oh Y, Herbst RS, Burris H, et al. Enzastaurin, an oral serine/threonine kinase inhibitor, as second- or third-line therapy of non-small-cell lung cancer. J Clin Oncol. 2008;26(7):1135-1141.

47. Camidge DR, Gail Eckhardt S, Gore L, et al. A phase I safety, tolerability, and pharmacokinetic study of enzastaurin combined with capecitabine in patients with advanced solid tumors. Anticancer Drugs. 2008;19(1):77-84

48. Spalding AC, Watson R, Davis ME, Kim AC, Lawrence TS, Ben-Josef E. Inhibition of protein kinase cbeta by enzastaurin enhances radiation cytotoxicity in pancreatic cancer. Clin Cancer Res. 2007;13(22 Pt 1): 6827-6833.

49. Rademaker-Lakhai JM, Beerepoot LV, Mehra N, et al. Phase I pharmacokinetic and pharmacodynamic study of the oral protein kinase $\mathrm{C}$ beta-inhibitor enzastaurin in combination with gemcitabine and cisplatin in patients with advanced cancer. Clin Cancer Res. 2007;13(15 Pt 1): 4474-4481.

50. Butowski N, Chang SM, Lamborn KR, et al. Phase II and pharmacogenomics study of enzastaurin plus temozolomide during and following radiation therapy in patients with newly diagnosed glioblastoma multiforme and gliosarcoma. Neuro Oncol. 2011;13(12): 1331-1338.
51. Usha L, Sill MW, Darcy KM, et al. A gynecologic oncology group phase II trial of the protein kinase C-beta inhibitor, enzastaurin and evaluation of markers with potential predictive and prognostic value in persistent or recurrent epithelial ovarian and primary peritoneal malignancies. Gynecol Oncol. 2011;121(3):455-461.

52. Rizvi MA, Ghias K, Davies KM, et al. Enzastaurin (LY317615), a protein kinase $\mathrm{C} \beta$ inhibitor, inhibits the AKT pathway and induces apoptosis in multiple myeloma cell lines. Mol Cancer Ther. 2006;5(7):1783-1789.

53. Moreau A, Jia X, Ngo HT, et al. Protein kinase C inhibitor enzastaurin induces in vitro and in vivo antitumor activity in Waldenström macroglobulinemia. Blood. 2007;109(11):4964-4972.

54. Ghobrial IM, Harousseau J, Treon SP, et al. Enzastaurin in previously treated Waldenstrom's macroglobulinemia: an open-label, multicenter, phase II study. ASH Annu Meet Abstr. 2009;114(22):3867.

55. Decouvelaere AV, Morschhauser F, Buob D, Copin MC, Dumontet C. Heterogeneity of protein kinase $\mathrm{C}$ beta(2) expression in lymphoid malignancies. Histopathology. 2007;50(5):561-566.

56. Morschhauser F, Seymour JF, Kluin-Nelemans HC, et al. A phase II study of enzastaurin, a protein kinase $\mathrm{C}$ beta inhibitor, in patients with relapsed or refractory mantle cell lymphoma. Ann Oncol. 2008;19(2):247-253.

57. Robertson MJ, Kahl BS, Vose JM, et al. Phase II study of enzastaurin, a protein kinase $\mathrm{C}$ beta inhibitor, in patients with relapsed or refractory diffuse large B-cell lymphoma. J Clin Oncol. 2007;25(13): 1741-1746.

58. Schwartzberg L, Hermann RC, Flinn IW, et al. Enzastaurin in patients with follicular lymphoma: results of a phase II study. ASCO Meet Abstr. 2010;28(15 Suppl):8040.

59. Querfeld C, Rizvi MA, Kuzel TM, et al. The selective protein kinase $\mathrm{C}$ beta inhibitor enzastaurin induces apoptosis in cutaneous T-cell lymphoma cell lines through the AKT pathway. J Invest Dermatol. 2006;126(7):1641-1647.

60. Forsyth CJ, Gomez D, Eliadis P, et al. Enzastaurin in patients with non-Hodgkin lymphomas: a multicenter, open-label, screening study. ASH Annu Meet Abstr. 2009;114(22):3719.

61. Querfeld C, Kuzel TM, Kim YH, et al. Multicenter phase II trial of enzastaurin in patients with relapsed or refractory advanced cutaneous T-cell lymphoma. Leuk Lymphoma. 2011;52(8):1474-1480.

62. Ysebaert L, Morschhauser F. Enzastaurin hydrochloride for lymphoma: reassessing the results of clinical trials in light of recent advances in the biology of B-cell malignancies. Expert Opin Investig Drugs. 2011;20(8):1167-1174.

63. Krejsgaard T, Kopp K, Ralfkiaer E, et al. A novel xenograft model of cutaneous T-cell lymphoma. Exp Dermatol. 2010;19(12): 1096-1102.

64. Doebbeling U. A mouse model for the Sézary syndrome. J Exp Clin Cancer Res. 2010;29:11.

65. Richards DA, Kuefler PR, Becerra C, et al. Gemcitabine plus enzastaurin or single-agent gemcitabine in locally advanced or metastatic pancreatic cancer: results of a phase II, randomized, noncomparative study. Invest New Drugs. 2011;29(1):144-153.

66. Pircher A, Hilbe W, Heidegger I, Drevs J, Tichelli A, Medinger M. Biomarkers in tumor angiogenesis and anti-angiogenic therapy. Int $J$ Mol Sci. 2011;12(10):7077-7099.

67. Paule B, Bastien L, Deslandes E, et al. Soluble isoforms of vascular endothelial growth factor are predictors of response to sunitinib in metastatic renal cell carcinomas. PLoS One. 2010;5(5):e10715.

68. Adjei AA. What is the right dose? The elusive optimal biologic dose in phase I clinical trials. J Clin Oncol. 2006;24(25):4054-4055.

69. Green LJ, Marder P, Ray C, et al. Development and validation of a drug activity biomarker that shows target inhibition in cancer patients receiving enzastaurin, a novel protein kinase C-beta inhibitor. Clin Cancer Res. 2006;12(11 Pt 1):3408-3415.

70. Rovedo MA, Krett NL, Rosen ST. Inhibition of glycogen synthase kinase-3 increases the cytotoxicity of enzastaurin. J Invest Dermatol. 2011;131(7):1442-1449. 
71. Baselga J, Campone M, Piccart M, et al. Everolimus in postmenopausal hormone-receptor-positive advanced breast cancer. $N$ Engl J Med. 2012;366(6):520-529.

72. Hanahan D, Weinberg RA. Hallmarks of cancer: the next generation. Cell. 2011;144(5):646-674.

73. Armstrong D, Ermisch S, Collins C, et al. A phase I study of enzastaurin and bevacizumab in patients with advanced cancer: response and clinical benefit in ovarian and other gynecologic cancers. Gynecol Oncol. 2010;116(3 Suppl 1):S16-S17.
74. Bodo J, Sedlak J, Maciejewski JP, Almasan A, Hsi ED. HDAC inhibitors potentiate the apoptotic effect of enzastaurin in lymphoma cells. Apoptosis. 2011;16(9):914-923.

75. Querfeld C, Guitart J, Kuzel TM, Rosen ST. Primary cutaneous lymphomas: a review with current treatment options. Blood Rev. 2003;17(3):131-142.

76. Hartford CM, Ratain MJ. Rapamycin: something old, something new, sometimes borrowed and now renewed. Clin Pharmacol Ther. 2007;82(4):381-388

\section{Publish your work in this journal}

Orphan Drugs: Research and Reviews is an international, peer-reviewed, open access journal publishing original research, reports, reviews and commentaries on all areas of the design and development of orphan drugs for the treatment of rare diseases through to clinical applications. Clinical outcomes, patient safety, and programs for the development and effective, safe, and sustained use of medicines will be a feature of the journal. The manuscript management system is completely online and includes a very quick and fair peer-review system, which is all easy to use. Visit http://www.dovepress.com/testimonials.php to read real quotes from published authors. 